\title{
N-ACETILCISTEÍNA - AÇÃO ANTIOXIDANTE E UTILIZAÇÃO NA CLÍNICA DE PEQUENOS ANIMAIS
}

\author{
Amanda Cristiane Ondani ${ }^{1}$, Marileda Bonafim Carvalho ${ }^{2}$, André Luiz Baptista \\ Galvão $^{2}$ \\ 1 Clínica São Francisco - Franca-SP \\ 2 Faculdade de Ciências Agrárias e Veterinárias - UNESP/Jaboticabal \\ Endereço para correspondência: Amanda Cristiane Ondani: ondani@gmail.com
}

RESUMO: A N-acetilcisteína, durante muito tempo, teve seu uso restrito à ação mucolítica e expectorante e ao tratamento da intoxicação por paracetamol/ acetaminofen. Com o advento de estudos acerca de radicais livres, os antioxidantes ganharam destaque e, dentre eles, a $\mathrm{N}$ acetilcisteína. Esta revisão de literatura tem por objetivo realçar a atividade farmacológica desse aminoácido em afecções da rotina clínica de pequenos animais, bem como apresentar as novas perspectivas de utilização em seres humanos, que poderão embasar, futuramente, estudos semelhantes em cães e gatos.

Palavras-chave: hiper-homocisteinemia; homocisteína; N-acetilcisteína, cão, gato.

\section{N-ACETYLCYSTEINE - ANTIOXIDANT ACTION AND USE IN THE SMALL ANIMAL CLINIC}

\begin{abstract}
For a long time, $\mathrm{N}$-acetylcysteine was only used as a mucolytic and as an expectorant agent in the treatment of paracetamol/ acetaminophen intoxication. With the advent of studies on free radicals, antioxidants are gaining prominence and the $\mathrm{N}$-acetylcysteine is amongst them. The objective of this article is to emphazise the farmacological activity of this amino acid in affections in the small animal clinical routine, as well as to show new perspectives of its use in humans, which could, in the future, form the base for similar studies in dogs and cats.
\end{abstract}

Key Words: homocystein; hyperhomocysteinemia; N-acetylcysteine; dog; cat. 


\section{INTRODUÇÃO}

A N-acetilcisteína é um aminoácido que, por muito tempo, foi utilizado como mucolítico e antídoto na intoxicação por paracetamol (Wilcke, 1984; Moldeus et al., 1986; Plumb, 2005). Atualmente, sua função antioxidante tem sido destacada em estudos acerca de substâncias reativas do metabolismo do oxigênio e radicais livres, como 0 peróxido de hidrogênio e o superóxido (Didoné et al., 2002; Miranda et al., 2004). Tais substâncias têm sido apontadas por alguns autores como responsáveis por lesões citotóxicas decorrentes do acúmulo de homocisteína, aminoácido metabólito da metionina, que além de produzi-las, diminui a atividade da glutationa peroxidase, um potente antioxidante (Loscalzo, 1996, Keaney e Loscalzo, 1997).

A presente revisão trata da utilização da $\mathrm{N}$-acetilcisteína na terapia de pequenos animais, explorando seu potencial antioxidante e abordando estudos em seres humanos e animais de laboratório que poderão, em um futuro próximo, embasar pesquisas em cães e gatos.

\section{DESENVOLVIMENTO}

\section{A $\mathrm{N}$-acetilcisteína e as substâncias reativas do metabolismo do oxigênio.}

A $\mathrm{N}$-acetilcisteína $\left(\mathrm{C}_{5} \mathrm{H}_{9} \mathrm{NO}_{3} \mathrm{~S}\right)$ é formada pelo aminoácido L-cisteína $\left(\mathrm{C}_{3} \mathrm{H}_{7} \mathrm{NO}_{2} \mathrm{~S}\right)$ adicionado de um grupo acetil $\left(-\mathrm{CO}-\mathrm{CH}_{3}\right)$, que torna mais rápida a absorção e distribuição da cisteína pela via oral (Figura 1). Sua indicação terapêutica, por muitos anos, ficou restrita à ação mucolítica e expectorante, bem como ao tratamento da intoxicação por paracetamol, tanto na Medicina quanto na Medicina Veterinária (Wilcke, 1984; Moldeus et al., 1986; Plumb, 2005).

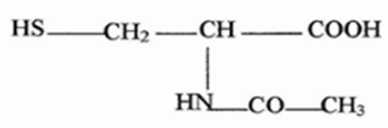

$\mathrm{N}$-acetilcisteína<smiles>NC(CS)C(=O)O</smiles>

Cisteína

Figura 1 - Estrutura química da $\mathrm{N}$-acetilcisteína e da cisteína.

A ação antioxidante da Nacetilcisteína deve-se à sua utilização como substrato para a síntese de glutationa peroxidase (cedendo a cisteína), um potente antioxidante, e ao átomo de hidrogênio presente no grupo tiol ou sulfidrila (-SH), que atua como um elétron na neutralização de radicais livres (Brunet et al.,1995; Di Mari et al., 1997; Didoné et al., 2002). Os radicais livres são átomos ou moléculas altamente reativos, com um número ímpar de elétrons em sua última camada eletrônica. De acordo com Ferreira e Matsubara (1997), o termo "radical livre" não é o mais conveniente para designar os agentes reativos patogênicos, uma vez que nem todos apresentam elétrons desemparelhados na última camada, como o peróxido de hidrogênio. Os autores sugerem 0 emprego de "espécies reativas do metabolismo do oxigênio" para designálos, já que em sua maioria são formados a partir do metabolismo do oxigênio. Quando há desequilíbrio entre antioxidantes e espécies reativas do metabolismo do oxigênio desenvolve-se o estresse oxidativo (Halliwell, 1993). Esta situação tem sido estudada em diversas afecções, muitas vezes associada à hiper-homocisteinemia (Starkebaum e Harlan, 1986; Lang et al., 2000).

\section{A homocisteína.}

A

$\left(\mathrm{HSCH}_{2} \mathrm{CH}_{2} \mathrm{CH}\left(\mathrm{NH}_{2}\right) \mathrm{CO}_{2} \mathrm{H}\right)$ é um 
aminoácido sulfurado, produto intermediário do metabolismo da metionina que, em altas concentrações, constitui um fator de risco para o desenvolvimento de doenças vasculares (Friedman et al., 2001). Quando a regulação da concentração de homocisteína intracelular é quebrada e o envio para o plasma está aumentado, ou o clearance de homocisteína plasmática está diminuído, desenvolvese a hiper-homocisteinemia (Guttormsen et al., 1994). A deficiência de vitaminas, principalmente ácido fólico e vitamina B12 que ocorre na insuficiência renal crônica (IRC), também contribui para o aumento da homocisteína por desfavorecer a formação de metionina a partir dela pela via da remetilação, que utiliza o primeiro como substrato e o segundo, como cofator (Refsum et al., 1998; Hoffer, 2006).

A produção de homocisteína ocorre naturalmente em todas as células, como consequência da conversão de metionina a cisteína (Friedman et al., 2001). Por ser uma substância tóxica às mesmas, levando à produção de espécies reativas do metabolismo do oxigênio, a homocisteína é exportada para o plasma quando a taxa de formação extrapola a capacidade de metabolização (Christensen et al., 1991; Selhub, 1999).

A metionina é um aminoácido adquirido diretamente da alimentação ou da quebra de proteínas. Dentro das células é convertida em S-adenosilmetionina, que é doadora de grupamentos metil para ácidos nucléicos, neurotransmissores, hormônios e fosfolipídios. Ao mesmo tempo, forma-se a S-adenosilhomocisteína, que é convertida em adenosina e homocisteína. Quando há excesso de metionina, a homocisteína é direcionada para a via da transulfuração, cuja primeira reação é catalisada pela enzima cistationina- $\beta$ - sintase (CBS), que é dependente de vitamina B6. Já em condições de balanço negativo de metionina, sua reconstituição se dá por duas vias. No fígado, uma grande proporção de homocisteína é remetilada pela betaínahomocisteína-metil-transferase (BHMT), que utiliza a betaína como doadora de metil. $\mathrm{Na}$ maioria dos tecidos, a remetilação da homocisteína é catalisada pela metionina sintase (MS), que utiliza a vitamina B12 como um cofator e folato como substrato (Finkelstein, 1990).

O grupo sulfidrila livre da Nacetilcisteína se liga preferencialmente aos receptores de cisteína e outros grupos sulfidrila das proteínas (incluindo da homocisteína), deslocando-os e formando $\mathrm{N}$-acetilcisteína-homocisteína ou $\mathrm{N}$-acetilcisteína-cisteína (Burgunder et al., 1989), o que proporciona a redução da concentração plasmática de homocisteína via clearance renal (Scholze et al., 2004). É importante notar que, em muitos processos patológicos, o fator desencadeante de hiper-homocisteinemia permanece desconhecido, mas a utilização da Nacetilcisteína tem sido associada à diminuição da homocisteína plasmática e, muitas vezes, melhora do quadro clínico do paciente (Tepel et al., 2003).

\section{Utilização da $\mathrm{N}$-acetilcisteína no trato respiratório.}

Tradicionalmente, a $\quad \mathrm{N}-$ acetilcisteína tem sido utilizada na Medicina Veterinária em afecções respiratórias e intoxicações por paracetamol. Nos felinos são comuns processos restritos ao trato respiratório superior, como as rinossinusites provocadas pelos herpesvírus e calicivírus no complexo respiratório. Esses vírus penetram pelas vias nasal, oral ou conjuntival, causando primariamente lise do epitélio nasal e acometendo, posteriormente, traquéia, 
brônquios e bronquíolos. As lesões são caracterizadas por necrose multifocal do epitélio, com infiltração neutrofílica e inflamação. A secreção nasal resultante dessas infecções por vezes é suficiente para o desenvolvimento de inapetência, agravando o quadro (Gaskell et al., 2007; Norsworthy, 2007). A Nacetilcisteína é usada como auxiliar no tratamento de doenças respiratórias devido à sua ação mucolítica. Quando administrada por nebulização, a $\mathrm{N}$ acetilcisteína diminui a viscosidade de secreções purulentas e não-purulentas, reduzindo as ligações dissulfídicas das mucoproteínas por meio do grupo sulfidrila livre (Church, 2006), porém, a administração por esta via tem sido apontada como causadora de hipersensibilidade e broncoespasmo (Plumb, 2005).

\section{Intoxicação por paracetamol/acetaminofen.}

O paracetamol ou acetaminofen é um analgésico e antipirético de uso humano extremamente tóxico para felinos (Boothe, 1990). Sinais clínicos de intoxicação ocorrem na dosagem de 50 a $60 \mathrm{mg} / \mathrm{kg}$, por via oral (Christiansen, 1980).

Na maioria das espécies animais, o paracetamol é metabolizado quando passa pela segunda vez no fígado, formando glicuronídeos e compostos ésteres sulfatados. Uma pequena porção é biotransformada por enzimas, na primeira passagem, a metabólitos tóxicos (Boothe, 1990). Estes são removidos pela conjugação com a glutationa antes que possam causar lesões no organismo. Nos gatos, em razão da deficiência ou concentrações extremamente baixas de algumas enzimas da família das glicuroniltransferases, há deficiência na conjugação de fármacos ou metabólitos da primeira passagem pelo fígado com o ácido glicurônico. Há, no entanto, vias alternativas, como a via da sulfatação. A conjugação dos metabólitos com sulfato é bem desenvolvida nos gatos, porém, ocorre rápida saturação dessa via após administração de doses elevadas de fármaco. A conjugação dos metabólitos com a glutationa é um mecanismo importante para a espécie, porém, também está sujeita à rápida depleção de suas reservas. Dessa forma, os metabólitos tóxicos da primeira fase $(\mathrm{N}$ acetil-p-benzoquinona) tendem a se acumular, causando lesão oxidativa à hemoglobina e aos hepatócitos (Wilcke, 1984; Boothe, 1990). Os sinais clínicos observados são vômitos, anorexia, mucosas pálidas, ptialismo, letargia e depressão (Boothe, 1990). A metemoglobinemia desenvolve-se rapidamente, além da formação de corpúsculos de Heinz e hemólise intravascular (Wilcke, 1984). Os gatos apresentam cianose, edema de face e membros, icterícia, hiperpneia e hipóxia, podendo vir a óbito. O quadro de cianose produzido pelo paracetamol é resultante da hipóxia decorrente da conversão de hemoglobina em metemoglobina, que não transporta oxigênio (Boothe, 1990). As alterações laboratoriais incluem metemoglobinemia, hiperbilirrubinemia, elevação da alanina-aminotransferase $(\mathrm{ALT})$ e aspartatoaminotransferase (AST), presença de corpúsculo de Heinz, reticulocitose, bilirrubinúria, hemoglobinúria e proteinúria (Wilcke, 1984). Neste caso, a N-acetilcisteína proporciona um substrato alternativo para conjugação com o metabólito tóxico do paracetamol, mantendo ou restaurando a concentração de glutationa. $\mathrm{O}$ tratamento com Nacetilcisteína é mais efetivo nas primeiras 8 a 16 horas após a ingestão do paracetamol e em cães e gatos, a dosagem recomendada em caso de intoxicação é de 130 a $140 \mathrm{mg} / \mathrm{kg}$ por via oral ou intravenosa inicialmente e após, $70 \mathrm{mg} / \mathrm{kg}$ a cada 4 ou 6 horas, por 
2 ou 3 dias (Grace, 2007; Webster e Cooper, 2009).

\section{Hepatopatias, insuficiência renal crônica e síndrome da imunodeficiência felina (FIV).}

Novas perspectivas terapêuticas da N-acetilcisteína incluem o tratamento de hepatopatias, insuficiência renal crônica (IRC) e infecções virais. A Nacetilcisteína é um fármaco capaz de repor os estoques celulares de glutationa, antioxidante fundamental no controle das lesões resultantes da reperfusão sanguínea, por exemplo. Como demonstrado por Didoné et al. (2002), em ratos submetidos à isquemia e reperfusão sanguínea hepática, a administração de N-acetilcisteína determinou diminuição significativa da congestão. Entretanto, não houve diferença quanto à presença de esteatose e necrose em relação ao grupo controle.

Webster e Cooper (2009) destacam a importância da utilização da $\mathrm{N}$-acetilcisteína na insuficiência hepática aguda causada por agentes tóxicos (carprofeno e trimetoprim em cães, diazepam e metimazole em gatos). Além disso, gatos com lipidose hepática severa podem se beneficiar do tratamento com este antioxidante que, ao aumentar a concentração de glutationa nas células, reduz a formação de corpúsculos de Heinz, condição comum nesta doença.

Pacientes humanos com IRC em estágio terminal apresentam alta mortalidade por motivos cardiovasculares (Tepel et al., 2003; Scholze et al., 2004). Essa condição acumula vários fatores de risco, tais como hipertensão, anemia, estresse oxidativo e concentração aumentada de homocisteína plasmática (Scholze et al., 2004). Estima-se que esse risco aumente $1 \%$ para cada micromol de aumento da concentração de homocisteína plasmática (Moustapha et al., 1998). A hiper-homocisteinemia tem se mostrado um fator de risco para o desenvolvimento de aterosclerose nas coronárias e vasos periféricos e cerebrais, bem como para o tromboembolismo arterial e venoso (Refsum et al., 1998). Hultberg et al. (1994) e Wiklund et al. (1996) verificaram que seres humanos tratados com $\mathrm{N}$-acetilcisteína, pelas vias oral ou intravenosa, apresentaram redução imediata e dose-dependente da homocisteína e cisteína plasmáticas. Deve-se evitar, no entanto, o uso de Nacetilcisteína em doses altas, em associação com suplementação de ferro (situação corriqueira na IRC, quando se utiliza a eritropoetina), devido à piora do estresse oxidativo pela formação de espécies reativas do metabolismo do oxigênio (Sprong et al., 1998).

Eck et al. (1989) verificaram que nos indivíduos infectados com HIV, as concentrações sanguíneas de grupos sulfidrila diminuem consideravelmente, e sugeriram uma possível associação entre 0 estresse oxidativo e o processo de apoptose na síndrome da imunodeficiência adquirida (AIDS). Além disso, Mórtola et al. (2000), utilizando cultivo celular de fibroblastos felinos cronicamente infectados com FIV, verificaram que 0 tratamento dessas células com $\mathrm{N}$-acetilcisteína e ácido ascórbico mostrou uma marcada inibição na produção da enzima transcriptase reversa. Estes resultados sugerem que a $\mathrm{N}$-acetilcisteína pode ser efetiva na manutenção da latência viral no cultivo celular infectado com FIV, porém, é necessário o uso contínuo do fármaco. O verdadeiro impacto destes tratamentos no prognóstico do gato infectado com FIV deverá ser avaliado por meio da utilização clínica dos fármacos testados (Mórtola et al., 2000). 


\section{CONCLUSÃO}

A N-acetilcisteína, como visto, constitui-se em um fármaco de utilização abrangente e promissora devido à ação antioxidante de seu grupo sulfidrila. Alguns estudos não confirmam seus benefícios, outros mostram resultados insatisfatórios ou indesejáveis. Porém, trabalhos que mostram bons resultados com sua utilização superam em número aqueles com resultados desfavoráveis.

Mais estudos na prática clínica com a $\mathrm{N}$-acetilcisteína devem ser incentivados, tanto em animais quanto em seres humanos, uma vez que resultados de experimentos controlados podem ser diferentes do que ocorre na realidade. Além disso, os animais podem servir como modelo experimental em algumas doenças similares às que ocorrem em seres humanos (como a síndrome da imunodeficiência felina e a AIDS), bem como estudos realizados em seres humanos podem ser extrapolados para a Medicina Veterinária, beneficiando também os animais.

\section{REFERÊNCIAS}

BOOTHE, D. M. Drug therapy in cats. A systems approach. Journal of the American Veterinary Medical Association, v.196, n.9, p.1502-1511, 1990.

BRUNET, J.; BOILY, M. J.; CORDEAU, S.; ROSIERS, C. Effects of $\mathrm{N}$-acetylcysteine in the rat heart reperfused after low-flow ischemia: evidence for a direct scavenging of hydroxyl radicals and a nitric oxide-dependent increase in coronary flow. Free Radical Biology and Medicine, v.19, n.5, p.627-638, 1995.

BURGUNDER, J. M; VARRIALE, A.; LAUTERBURG, B.H. Effect of N-acetylcysteine on plasma cysteine and glutathione following paracetamol administration. European Journal of Clinical Pharmacology, v.36, n.2, p.127$131,1989$.
CHRISTENSEN, B.; REFSUM, H.; VINTERMYR, O.; UELAND, P. M. Homocysteine export from cells cultured in the presence of physiological or superfluous levels of mehionine: methionine loading of non-transformed, transformed, proliferation, and quiescent cells in culture. Journal of Cellular Physiology, v.146, n.1, p.52, 1991.

CHRISTIANSEN, G. The toxicity of selected therapeutic agents used in cats. Veterinary Medicine/Small Animal Clinician, v.75, n.7, p.1133-1141, 1980.

$\mathrm{CHURCH}, \mathrm{D}$. Drugs used in the management of respiratory diseases. In: WORLD CONGRESS WSAVA/FECAVA/CSAVA, 2006, Praga. Proceedings... Praga: World Small Animal Veterinary Association, p.167-172, 2006.

DIDONÉ, E. C.; CERSKI, C. T.; KALIL, A. N. Nacetilcisteína diminui a congestão hepática na lesão de isquemia e reperfusão - estudo experimental. Revista do Colégio Brasileiro de Cirurgiões, v.29, n.4, p.191-196, 2002.

DI MARI, J.; MEGYESI, J.; UDVARHELYI, N.; PRICE, P.; DAVIS R.; SAFIRSTEIN, R. N-acetyl cysteine ameliorates ischemic renal failure. American Journal of Physiology - Renal Physiology, v.272, n.3, p.F292-F298, 1997.

ECK, H. P.; GMUNDER, H.; HARTMANN, M.; PETZOLDT, D.; DANIEL, V.; DROGE, W. Low concentrations of acid-soluble thiol (cysteine) in the blood plasma of HIV-1-infected patients. Biological Chemistry Hoppe-Seyler, v.370, n.2, p.101-108, 1989.

FERREIRA, A. L. A.; MATSUBARA, L. S. Radicais livres: conceitos, doenças relacionadas, sistema de defesa e estresse oxidativo. Revista da Associação Médica Brasileira, v.43, n.1, p.61-68, 1997.

FINKELSTEIN, J. D. Methionine metabolism in mammals. The Journal of Nutritional Biochemistry, v.1, p.228-237, 1990.

FRIEDMAN, A. N.; BOSTOM, A. G.; SELHUB, J.; LEVEY, A. S.; ROSENBERG, I. H. The kidney and homocysteine metabolism. Journal of the American Society of Nephrology, v.12, n.10, p.2181-2189, 2001. 
GASKELL, R.; DAWSON, S.; RADFORD, A.; THIRY, E. Feline herpesvirus. Veterinary Research, v.38, n.2, p.337-354, 2007.

GRACE, S. F. Acetaminophen toxicosis. In: NORSWORTHY, G. D.; CRYSTAL, M. A.; GRACE, S. F.; TILLEY, L. P. The Feline Patient. $3^{\text {rd }}$ ed. Ames: Blackwell Publishing, 2007, Cap.1, p.5-6.

GUTTORMSEN, A. B.; SCHNEEDE, J.; FISKERSTRAND, T. UELAND, P. M.; REFSUM, $\mathrm{H}$. M. Plasma concentrations of homocysteine and other aminothiol compounds are related to food intake in healthy subjects. The Journal of Nutrition, v.124, n.10, p.1934-1941, 1994.

HALLIWELL, B. The role of oxygen radicals in human disease, with particular reference to the vascular system. Haemostasis, v.23, n.S1, p.118-126, 1993.

HOFFER, L. J. Testing the homocysteine hypothesis in end-stage renal disease: problems and possible solutions. Kidney International, v.69, n.9; p.1507-1510, 2006.

HULTBERG, B.; ANDERSSON, A.; MASSON, P.; LARSON, M.; TUNEK, A. Plasma homocysteine and thiol compound fractions after oral administration of $\mathrm{N}$-acetylcysteine. Scandinavian Journal of Clinical and Laboratory Investigation, v.54, n.6, p.417-422, 1994.

KEANEY, J. F.; LOSCALZO, J. Homocyst(e)ine decreases bioavailable nitric oxide by a mechanism involving glutathione peroxidase. Journal of Biological Chemistry, v.272, n.27, p.17012-17017, 1997.

LANG, D.; KREDAN, M. B.; MOAT, S. J.; HUSSAIN, S. A.; POWELL, C. A.; BELLAMY, M. F.; POWERS, H. J.; LEWIS, M. J. Homocysteine-induced inhibition of endotheliumdependent relaxation in rabbit aorta: role for superoxide anions. Arteriosclerosis, Thrombosis and Vascular Biology, v.20, n.2, p.422-427, 2000.

LOSCALZO, J. The oxidant stress of hyperhomocyst(e)inemia. The Journal of Clinical Investigation, v.98, n.1, p.5-7, 1996.

MIRANDA, L. E. C.; VIARO, F.;CENEVIVA, R.; EVORA, P. R. B. As bases experimentais da lesão por isquemia e reperfusão do fígado. Revisão. Acta Cirúrgica Brasileira, v.19, n.1, p.3-12, 2004.

MOLDEUS, P.; COTGREAVE, I. A.; BERGREN, $M$. Lung protection by a thiol-containing antioxidant: N-acetylcysteine. Respiration, v.50, n.1, p.31-42, 1986.

MÓRTOLA, E.; ENDO, Y.; RISSO, M; GIMENO, E. Estudio comparativo de los efectos antivirales de agentes antioxidantes: N-acetilcisteína y ácido ascórbico e inmunosupresores: ciclosporina A y tacrolimus em células crónicamente infectadas con El virus de la inmunodeficiencia felina. Veterinaria México, v.31, n.4, p.271-277, 2000.

MOUSTAPHA, A.; NASO, A.; NAHLAWI, M.; GUPTA, A.; ARHEART, K. L.; JACOBSEN, D. W.; ROBINSON, K.; DENNIS, V. W. Prospective study of hyperhomocysteinemia as an adverse cardiovascular risk factor in end-stage renal disease. Circulation, v.97, n.2, p.138-141, 1998.

NORSWORTHY, G. D. Nasal and frontal sinus infections. In: NORSWORTHY, G. D.; CRYSTAL, M. A.; GRACE, S. F.; TILLEY, L. P. The Feline Patient. $3^{\text {rd }}$ ed. Ames: Blackwell Publishing, 2007, Cap.92, p.200-202.

PLUMB, D. C. Acetylcysteine. In:_. Plumb's Veterinary Drug Handbook. 5.ed. Ames: Blackwell Publishing, 2005. p.9-10.

REFSUM, H.; UELAND, P. M.; NYGÅRD, O.; VOLLSET, E. Homocysteine and cardiovascular disease. Annual Review of Medicine, v.49, p.31-62, 1998.

SCHOLZE, A.; RINDER, C.; BEIGE, J.; RIEZLER, R.; ZIDEK, W.; TEPEL, M. Acetylcysteine reduces plasma homocysteine concentration and improves pulse pressure and endothelial function in patients with end-stage renal failure. Circulation, v.109, n.3, p.369-374, 2004.

SELHUB, J. Homocysteine metabolism. Annual Review of Nutrition, v.19, p.217-246, 1999.

SPRONG, R. C.; WINKELHUYZEN-JANSSEN, A. M. L.; AARSMAN, C. J. M.; VAN OIRSCHOT, J. F. L. M.; VAN DER BRUGGEN, T.; VAN 
ASBECK, B. S. Low-dose $\mathrm{N}$-acetylcysteine protects rats against endotoxin-mediated oxidative stress, but high-dose increases mortality. American Journal of Respiratory and Critical Care Medicine, v.157, n.4, p.12831293, 1998.

STARKEBAUM, G; HARLAN, J. M. Endothelial cell injury due to copper-catalyzed hydrogen peroxide generation from homocysteine. The Journal of Clinical Investigation, v.77, n.4, p.1370-1376, 1986.

TEPEL, M.; VAN DER GIET, M.; STATZ, M.; JANKOWSKI, J.; ZIDEK, W. The antioxidant acetylcysteine reduces cardiovascular events in patients with end-stage renal failure: a randomized, controlled trial. Circulation, v.107, n.7, p.992-995, 2003.

WEBSTER, C. R. L; COOPER, J. Therapeutic use of cytoprotective agents in canine and feline hepatobiliary disease. Veterinary Clinics of North America: Small Animal Practice, v. 39, n. 3, p. $631-652,2009$.

WIKLUND, O.; FAGER, G.; ANDERSSON, A.; LUNDSTAM, U.; MASSON, P.; HULTBERG, B. $\mathrm{N}$-acetylcysteine treatment lowers plasma homocysteine but not serum lipoprotein (a) levels. Atherosclerosis, v.119, n.1, p.99-106, 1996.

WILCKE, J. R. Idiosyncracies of drug metabolism in cats. Effects on pharmacotherapeutics in feline practice. The Veterinary Clinics of North America: Small Animal Practice, v.14, n.6, p.1345-1354, 1984. 